\title{
Canadian Adalimumab Postmarketing Observational Epidemiological Study Assessing Effectiveness in Psoriatic Arthritis (COMPLETE-PsA): 12-month Results of Comparative Effectiveness of Adalimumab and nbDMARDs
}

\author{
Majed M. Khraishi ${ }^{1}$, Valencia P. Remple ${ }^{2}$, Samuel Silverberg ${ }^{3}$, Jacqueline C. Stewart ${ }^{4}$, Brandusa Florica ${ }^{5}$, \\ and Louis Bessette ${ }^{6}$
}

\begin{abstract}
Objective. COMPLETE-PsA was an observational study of biologic-naïve Canadian adults with active psoriatic arthritis (PsA) treated with adalimumab (ADA) or a nonbiologic disease-modifying antirheumatic drug (nbDMARD) regimen, after inadequate response/intolerance to a current nbDMARD treatment regimen. The aim of this analysis was to assess the 12-month effectiveness of ADA vs nbDMARDs.

Methods. Patients enrolled between March 2012 and November 2017 were included. The following clinical variables and patient-reported outcomes were collected/calculated per routine care: Disease Activity Index for Psoriatic Arthritis in 28 joints (DAPSA28), Disease Activity Score in 28 joints (DAS28), erythrocyte sedimentation rate (ESR), C-reactive protein, physician global assessment (PGA), patient global assessment (PtGA), pain, Health Assessment Questionnaire-Disability Index (HAQ-DI), 12-item Short Form Health Survey, enthesitis, dactylitis, body surface area (BSA), and time to achieving American College of Rheumatology (ACR) 50, ACR70, and modified minimal disease activity (mMDA).

Results. Two hundred and seventy-seven ADA-treated and 148 nbDMARD-treated patients were included. At baseline, ADA-treated patients were less likely to be employed, had longer morning stiffness, higher DAPSA28, DAS28, PGA, PtGA, pain, and HAQ-DI, and lower prevalence of dactylitis $($ all $P<0.05)$. ADA-treated patients showed lower baseline-adjusted DAPSA28 (16.5 vs 26.6), DAS28 (2.8 vs 3.9), PGA (25.3 vs 37.1), and ESR (10.4 vs $15.0 \mathrm{~mm} / \mathrm{h}$ ) after 3 months compared to nbDMARD-treated patients, with observed improvements maintained to month 12. Time to achievement of ACR50, ACR70, and mMDA was significantly shorter $(P<0.001)$ among ADA-treated patients, with the likelihood of having dactylitis (odds ratio [OR] 0.4, 95\% CI 0.2-0.6) and BSA<3\% (OR 2.7, 95\% CI 1.5-5.0) significantly lower and higher, respectively. Switching to another biologic was less likely in ADA-treated vs nbDMARD-treated patients (hazard ratio $0.3,95 \%$ CI $0.2-0.5$ ).

Conclusion. In a real-world Canadian population of patients with PsA, ADA was more effective than nbDMARDs at reducing disease activity and the severity of skin involvement, and demonstrated higher retention. [ClinicalTrials.gov: NCT01559038]
\end{abstract}

Key Indexing Terms: disease-modifying antirheumatic drugs, longitudinal studies, nonsteroidal antiinflammatory drugs, psoriatic arthritis

The design, study conduct, and financial support for the study were provided by AbbVie.

${ }^{I}$ M.M. Khraishi, MB BCh, FRCP(C), Memorial University of Newfoundland, St. John's, Newfoundland and Labrador, Canada; ${ }^{2}$ V.P. Remple, MSN, PhD, AbbVie Biopharmaceuticals Sarl, Rungis, France; ${ }^{3} S$. Silverberg, MD, Etobicoke General Hospital, Toronto, Ontario, Canada; ${ }^{4}$ J.C. Stewart, MD, FRCPC, University of British Columbia, Penticton, British Columbia, Canada; ${ }^{5}$ B. Florica, $M D, P h D, F R C P C$, University of Toronto, Toronto, Ontario, Canada $;{ }^{6} L$. Bessette, MD, FRCPC, MSc, Laval University, Centre Hospitalier de l'Université Laval, Quebec City, Quebec, Canada.

$M M K$ is a speaker, consultant, and principal investigator for AbbVie. VPR receives a salary and stock options as an employee of AbbVie. SS is an advisory board consultant for AbbVie and a consultant for Janssen. JCS is an advisory
board consultant for AbbVie, Amgen, Celgene, Merck, Novartis, Pfizer, Sanofi Genzyme, Celltrion, Roche, and Sandoz; a speaker for AbbVie, Amgen, Janssen, and Eli Lilly; and has participated in research with AbbVie, BMS, Janssen, and Roche. BF is a speaker for Merck, AbbVie, Roche, BMS, and Novartis; a consultant for Roche, AbbVie, Pfizer, Janssen, Celgene, and UCB; and an investigator for AbbVie, Pfizer, and BMS. $L B$ is a speaker for Amgen, BMS, Janssen, Roche, UCB, AbbVie, Pfizer, Merck, Celgene, Eli Lilly, and Novartis; a consultant for Amgen, BMS, Janssen, Roche, UCB, AbbVie, Pfizer, Celgene, Eli Lilly, and Novartis; and an investigator for Amgen, BMS, Janssen, Roche, UCB, AbbVie, Pfizer, Merck, Celgene, Sanofi, Eli Lilly, and Novartis.
Address correspondence to Dr. M.M. Khraishi, 220 Elizabeth Ave, St. John's, NLA1C 5S7, Canada.Email:mkbraish@mun.ca.
Accepted for publication December 24, 2021. 
Psoriatic arthritis (PsA) is an autoimmune inflammatory spondylarthritis characterized by psoriasis $(\mathrm{PsO})$ and arthritis. ${ }^{1,2,3}$ Symptoms and clinical findings associated with PsA include enthesitis, inflammatory back pain, stiffness, dactylitis, swollen/ tender/painful joints, scaly and itchy patches on the skin, nail lesions, and extraarticular manifestations. The onset of skin and arthritis manifestations typically is not simultaneous, with $\mathrm{Ps} \mathrm{O}$ preceding the arthritis by up to 15 years in the majority (83-87\%) of patients. ${ }^{1,2}$ Further, both peripheral and axial arthritis may be present in patients with PsA.

The American College of Rheumatology $(\mathrm{ACR})^{4}$ and the Group for Research and Assessment of Psoriasis and Psoriatic Arthritis ${ }^{5}$ recommendations for management of PsA advocate for the use of nonbiologic disease-modifying antirheumatic drugs (nbDMARDs), such as methotrexate (MTX) and sulfasalazine, as first-line therapy for peripheral articular manifestations, and for the use of nonsteroidal antiinflammatory drugs (NSAIDs) when there is axial involvement. Second-line therapy includes the use of biologics, such as tumor necrosis factor (TNF) inhibitors, which are used to treat peripheral articular manifestations when initial therapies are ineffective.

ADA was first approved in Canada for the treatment of active PsA in $2007,{ }^{6}$ to reduce the signs and symptoms of arthritis, and to prevent the progression of structural damage, while improving physical function. Clinical trials have previously demonstrated that ADA is effective at reducing the signs and symptoms of both PsA and $\mathrm{PsO}$ and are well tolerated, with a safety profile comparable to placebo groups. ${ }^{7,8,9,10,11,12}$ Real-world studies have also established $\mathrm{ADA}$ as being effective and well tolerated in PsA and PsO. ${ }^{13,14,15,16,17}$

Although the efficacy of ADA has been established in clinical trials, comparative effectiveness studies are limited. The aim of this study was to assess the real-world effectiveness of ADA compared to nbDMARDs for the management of articular and dermatological manifestations, and their effect on patient quality of life (QOL) over 12 months of treatment among biologic-naive patients with moderate to severe PsA during routine care in Canada.

\section{METHODS}

Study design. COMPLETE-PsA was a prospective, observational, cohort study among patients enrolled from the offices of community rheumatologists across Canada between March 2012 and November 2017. Patients were followed up for a period of up to 2 years, with recommended visits at 3 , $6,12,18$, and 24 months, and all clinical decisions were based on the physicians' judgment, regional regulations, and the respective product monographs (ClinicalTrials.gov: NCT01559038).

Analysis population. Eligible patients were aged $\geq 18$ years with active PsA, defined as $\geq 3$ tender and swollen joints, along with active psoriatic skin lesions or a confirmed history of PsO. Patients were required to have shown inadequate response or intolerance to their current nbDMARD regimen and, as per the opinion of their treating physician, required a change in treatment. Patients were excluded from the study if they had been treated previously with a biologic agent, were participating in other prospective studies, or were considered by the treating physician to have a condition prohibiting them from participating or obscuring the assessment of their PsA treatment.

All analyses were performed on patients who signed the informed consent, met the inclusion and exclusion criteria, and received at least 1 dose of study medication within 30 days from baseline. Although patients were analyzed according to their baseline treatment group, patients who switched treatment during the follow-up period, defined as starting a new (ADA group) or first (nbDMARD group) biologic, did not contribute data after their switch date.

Data collection. The following patient demographic and disease characteristics were collected at baseline as per routine care: age, sex, race, employment status, disease duration, family history, rheumatoid factor status, dominant subtype of PsA, and past history of nbDMARD use. Clinical variables assessed over time included duration of morning stiffness, BSA with $\mathrm{PsO}$, enthesitis (proximal Achilles tendon and/or plantar fascia; assessed as present/absent), dactylitis (swelling graded as mild/moderate/ severe for each affected digit), physician global assessment (PGA; visual analog scale [VAS] 0-100), erythrocyte sedimentation rate (ESR), and $\mathrm{C}$-reactive protein (CRP). Patient-reported outcomes (PROs) collected over time were the patient global assessment (PtGA; VAS 0-100), pain (VAS 0-100), the Health Assessment Questionnaire-Disability Index (HAQ-DI), and the 12-item Short Form Health Survey (SF-12; not assessed at 3 months).

Study endpoints. At the time of study design, the primary effectiveness endpoint was the change over time in the Disease Activity Score in 28 joints (DAS28). At the time of analysis, Disease Activity Index for Psoriatic Arthritis in 28 joints (DAPSA28) was also calculated as a key endpoint. ${ }^{18}$

Secondary effectiveness endpoints included the following: changes over time in ESR, CRP, PGA, PtGA, pain, HAQ-DI, and SF-12 mental component summary (MCS) and physical component summary (PCS) scores; presence/absence of enthesitis and dactylitis; achievement of $\mathrm{PsO}$ body surface area (BSA) $\leq 3 \% ; 50 \%$ or $70 \%$ improvement in ACR criteria (ACR50/70); and achievement of modified minimal disease activity (mMDA; defined as achievement of 5 out of 7 of the following measures: tender joint count in 28 joints [TJC28] and swollen joint count in 28 joints $[\mathrm{SJC} 28] \leq 1$ each, $\mathrm{BSA} \leq 3 \%$, pain VAS $\leq 15, \mathrm{PtGA} \leq 20$, HAQ-DI $\leq 0.5$, and absence of enthesitis).

Safety was ascertained through the incidence of treatment-emergent serious adverse events (SAEs).

Statistical analyses. Baseline patient demographics and characteristics were assessed using summary statistics, including the mean and SD for continuous variables, and the counts and proportions for categorical variables. Differences between treatment groups (ADA vs nbDMARD) were assessed for statistical significance using the independent-samples $t$ test for continuous variables and the chi-square or Fisher exact test, as appropriate, for categorical variables.

The changes in DAS28 and DAPSA28 over 12 months of treatment were compared between treatment groups using a linear mixed model with repeated measures adjusting for baseline levels of the respective outcome; a similar approach was used to assess between-group differences with respect to ESR, CRP, PGA, PtGA, pain, HAQ-DI, and SF-12 MCS and PCS scores. As a sensitivity analysis, these models were repeated by additionally adjusting for potential confounders, defined as baseline characteristics with a $P \leq 0.1$ in the between-group comparison.

For evaluation of the presence of enthesitis and dactylitis as well as achievement of BSA $<3 \%$ over time, generalized estimating equations (GEE) models with repeated measures were used, adjusting for baseline levels of the respective outcome. A sensitivity analysis adjusting for potential confounders was also conducted as described above.

Time to treatment switch, as well as time to achieving ACR50, ACR70, and $\mathrm{mMDA}$ was assessed using Kaplan-Meier survival analysis. In addition, a sensitivity analysis adjusting for baseline SJC28/TJC28, baseline HAQ-DI (only for mMDA), and potential confounders, defined as described above, was also conducted for ACR50, ACR70, and mMDA using Cox regression.

Treatment-emergent SAEs were coded using Medical Dictionary for Regulatory Activities (MedDRA) version 13.1 and were described by system organ class and preferred term. Analyses were conducted using SAS software, version 9.4 (SAS Institute) 
Handling of missing data. Missing responses on the SF-12 questionnaire items were imputed per the SF-12 scoring manual. For all other outcome measures, no imputations were made for missing values that were handled using mixed models with repeated measures, GEE models, and right censoring in the case of survival analyses.

Ethics approval. Ethics approval was obtained from Advarra (formerly Institutional Review Board Services) for private practice sites. Local institutional review boards included the following: the University of British Columbia Clinical Research Ethics Board (ref no.: H12-03561), Comité d'éthique de la recherche en santé chez l'humain du Centre Hospitalier Universitaire de Sherbrooke, Health Research Ethics Authority (St. Clare's Mercy Hospital, ref no.: 12.072), and Capital Health Research Ethics Board (Nova Scotia Health Authority, REB no.: CDHA-RS/2012-303).

Prior to inclusion in the study, all patients were required to sign a written informed consent to allow use of their data in the study. Since this was not an interventional study and patients were treated as per routine care, the purpose of the informed consent was to allow the use of the data and communication with the patients by study personnel, as required.

\section{RESULTS}

A total of 277 patients treated with ADA and 148 patients treated with nbDMARDs were included. At baseline, patients in the ADA group were less likely to be employed, had longer morning stiffness, and had significantly higher DAPSA28, DAS28, TJC28, SJC28, PGA, PtGA, pain, and HAQ-DI scores compared to the nbDMARD group (Table 1). The prevalence of dactylitis, however, was lower at baseline among ADA-treated patients. At baseline, $80.1 \%$ of patients in the ADA group were taking nbDMARDs, and $43.7 \%$ and $55.4 \%$ of patients were taking NSAIDs in the ADA and nbDMARD groups, respectively (Table 1). The types of nbDMARDs taken at baseline are shown in Table 1. Other baseline characteristics were comparable between groups. When assessing disease activity indexes over time, both DAPSA28 and DAS28 were significantly different $(P<0.001)$ between treatment groups, with lower baseline-adjusted scores observed among ADA-treated patients as early as month 3 (DAPSA28 16.5, 95\% CI 14.2-18.8 vs 26.6, 95\% CI 22.9-30.2; DAS28 2.8, 95\% CI 2.6-3.0 vs 3.9, 95\% CI 3.6-4.1) and maintained until month 12 (Figure 1A,B). Similar significant differences over time were observed in favor of ADA for ESR, CRP, and PGA (Figures 1C-E). In terms of PROs, upon adjusting for baseline levels, ADA-treated patients reported significantly lower PtGA, HAQ-DI, and pain over the 12-month follow-up (Figure 2A-C). Further, SF-12 PCS was significantly higher among patients in the ADA group at 6, but not 12, months (Figure 2D). No differences were observed between groups in terms of SF-12 MCS (Figure 2E). Further adjustment for potential confounders had no effect on the aforementioned findings, showing similar results in favor of ADA (Supplementary Figures 1 and 2, available with the online version of this article).

Based on GEE models adjusting for baseline presence of enthesitis, the odds of having enthesitis over time were statistically comparable between treatment groups (odds ratio [OR] $0.7,95 \%$ CI 0.4-1.2); however, the odds of having dactylitis were significantly lower among ADA-treated patients (OR 0.4, 95\% CI 0.2-0.6; Table 2). Further, patients in the ADA group were more likely to have a BSA $\leq 3 \%$ over time (OR 2.7,
95\% CI 1.5-5.0). Adjustment for potential confounders had no effect on the aforementioned findings, showing similar results in favor of ADA (Supplementary Table 1, available with the online version of this article).

Time to achieving clinical effectiveness endpoints was significantly shorter $(P<0.001)$ in ADA-treated compared to nbDMARD-treated patients, with corresponding hazard ratios (HRs) of 2.29 (95\% CI 1.55-3.40), 2.92 (95\% CI 1.70-5.01), and 2.11 (95\% CI 1.47-3.02), respectively, for ACR50, ACR70, and mMDA (Figure $3 \mathrm{~A}-\mathrm{C}$ ). The cumulative probabilities of achieving each outcome at 3,6 , and 12 months in each treatment group are also shown in Figure 3A,B,C, indicating significant differences after as early as 3 months of treatment. Adjustment for potential confounders had no effect on the aforementioned findings, showing similar results in favor of ADA (Supplementary Table 2, available with the online version of this article).

Over a mean (SD) follow-up of 9.7 (2.9) vs 8.6 (3.8) months, ADA-treated patients were less likely to switch biologics compared to nbDMARD-treated patients initiating a biologic (HR 0.3, 95\% CI 0.2-0.5). More specifically, the 12-month survival probability was $85.8 \%$ (95\% CI 79.8-90.1\%) vs $63.4 \%$ (95\% CI 53.2-72.0\%) for ADA vs nbDMARD-treated patients (Figure 4). In terms of study retention, $8.3 \%(n=23)$ of patients in the ADA group vs $16.2 \%(\mathrm{n}=24)$ of those in the nbDMARD group were discontinued due to loss to follow-up (1.8\% vs $5.4 \%$ ), withdrawal of consent ( $2.5 \%$ vs $2.7 \%$ ), and other reasons ( $4 \%$ vs $8.1 \%)$, respectively.

SAEs were reported for 15 (5.4\%) patients in the ADA group. The most common SAE types were cerebrovascular accident $(\mathrm{n}=2$; both likely not related to ADA per the treating physician) and pneumonia ( $\mathrm{n}=2 ; 1$ likely related to ADA and 1 possibly related to ADA). The full list of SAEs is included in Supplementary Table 3 (available with the online version of this article). One patient in the nbDMARD group experienced a SAE (rectal fissure).

\section{DISCUSSION}

Although previous studies have demonstrated the efficacy of ADA for the treatment of PsA, there are limited real-world data comparing ADA to nbDMARDs, with only 1 , to our knowledge, postapproval registry study assessing the 6-month comparative effectiveness of pooled anti-TNFs vs MTX. ${ }^{19}$ Similar to our study, the authors showed that patients treated with anti-TNF inhibitors presented with more severe baseline disease as well as experienced significantly greater improvements in disease activity, function, and QOL. ${ }^{19}$

In the current study, compared to nbDMARDs, treatment with ADA was associated with significantly greater improvements in acute-phase reactants, pain, PtGA, PGA, and composite indexes as early as 3 months, indicating a more rapid reduction of inflammation. Time to achieving ACR20, ACR50, and mMDA was also significantly shorter among ADA-treated patients. The observed rates of ACR50 and ACR70 were comparable or higher to those reported in the 12-week ACCLAIM ${ }^{20}$ and STEREO $^{21}$ observational studies; however, these rates may not be directly comparable because of differences in the populations 


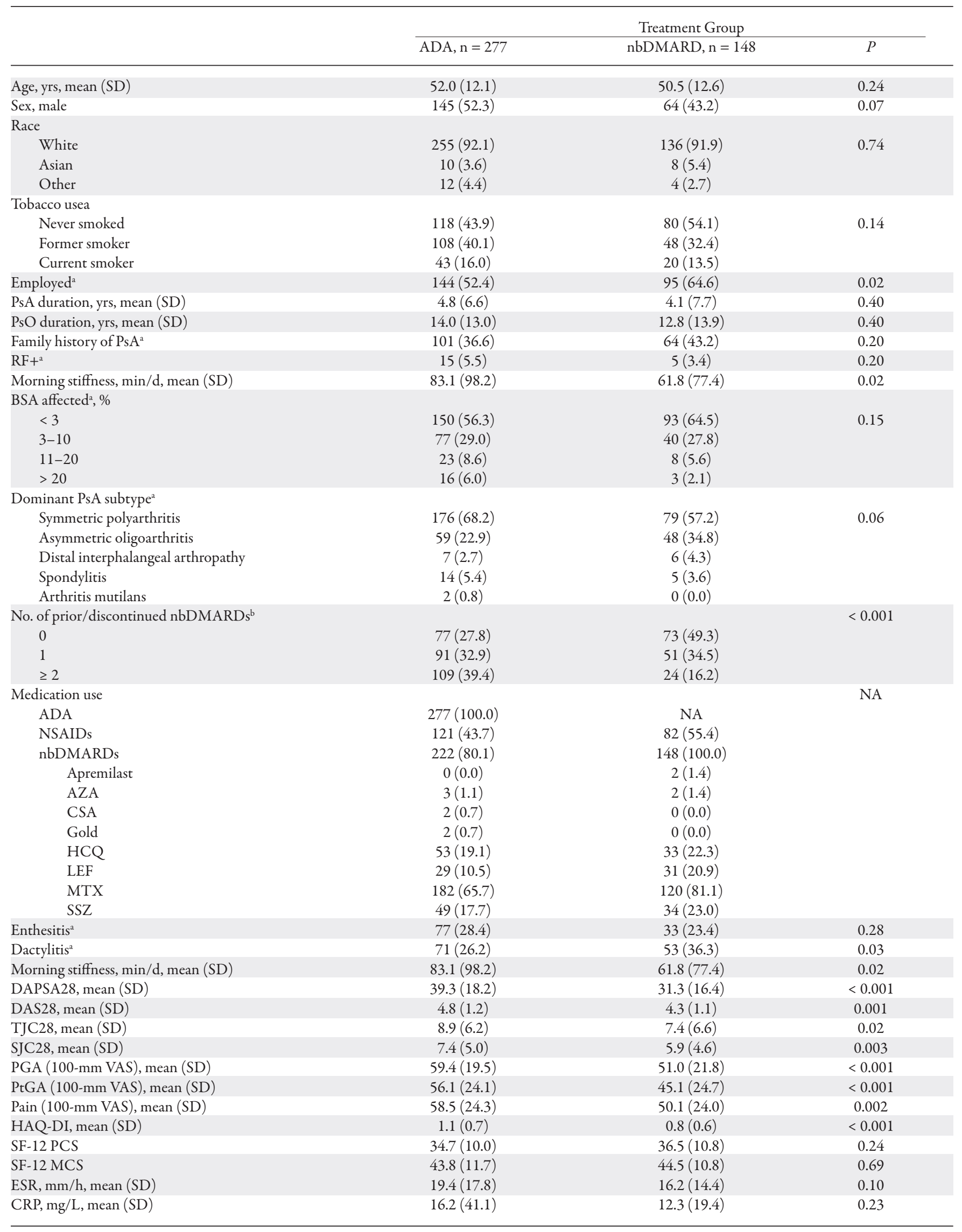


Values are $\mathrm{n}(\%)$ unless otherwise indicated. ${ }^{a}$ Proportions based on patients with available data. Missing or unknown information for ADA- and $\mathrm{nbDMARD}$-treated patients, respectively, were as follows: tobacco use $(\mathrm{n}=8$ and $\mathrm{n}=0)$, employment status $(\mathrm{n}=2$ and $\mathrm{n}=1)$ family history of PsA ( $\mathrm{n}=1$ and $n=0)$, RF status ( $n=3$ and $n=0)$, BSA $(n=11$ and $n=4)$, dominant PsA subtype $(n=19$ and $n=10)$, enthesitis $(n=6$ and $n=7)$, dactylitis $(n=6$ and $n=2)$, morning stiffness $(n=12$ and $n=7)$, DAPSA28 $(n=65$ and $n=44)$, DAS28 ( $n=49$ and $n=33)$, TJC28 ( $n=2$ and $n=0)$, SJC28 ( $n=2$ and $n=1)$, PGA $(\mathrm{n}=15$ and $\mathrm{n}=23)$, PtGA $(\mathrm{n}=33$ and $\mathrm{n}=23)$, pain $(\mathrm{n}=35$ and $\mathrm{n}=25)$, HAQ-DI $(\mathrm{n}=34$ and $\mathrm{n}=23)$, SF-12 $(\mathrm{n}=148$ and $\mathrm{n}=83)$, ESR $(\mathrm{n}=87$ and $\mathrm{n}=49)$, and CRP $(\mathrm{n}=36$ and $\mathrm{n}=23) .{ }^{\mathrm{b}}$ Excluding nbDMARDs initiated prior to baseline that were ongoing at baseline. ADA: adalimumab; AZA: azathioprine; BSA: body surface area; CRP: C-reactive protein; CSA: cyclosporine A; DAPSA: Disease Activity Index for Psoriatic Arthritis in 28 joints; DAS28: Disease Activity Score in 28 joints; ESR: erythrocyte sedimentation rate; HAQ-DI: Health Assessment Questionnaire-Disability Index; HCQ: hydroxychloroquine; LEF: leflunomide; MCS: mental component summary; PGA: physician global assessment; MTX: methotrexate; NA: not applicable; NSAID: nonsteroidal antiinflammatory drug; nbDMARD: nonbiologic disease-modifying antirheumatic drug; PCS: physical component summary; PsA: psoriatic arthritis; PsO: psoriasis; PtGA: patient global assessment; RF: rheumatoid factor; SF-12: 12-item Short Form Health Survey; SJC28: swollen joint count in 28 joints; SSZ: sulfasalazine; TJC28: tender joint count in 28 joints; VAS: visual analog scale.

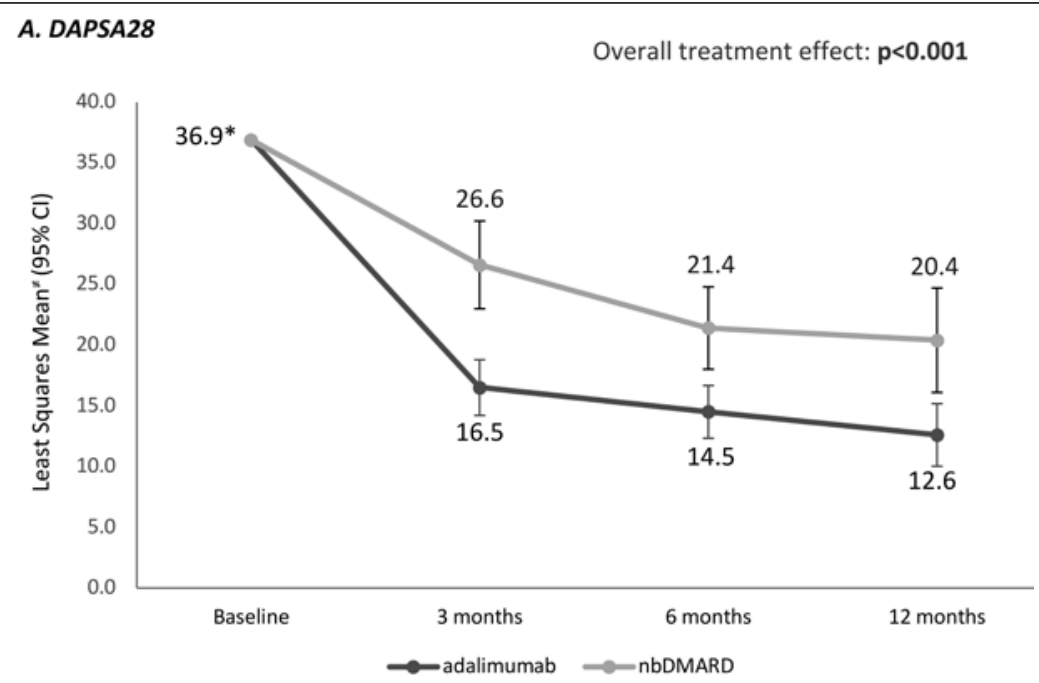

\section{B. DAS28}

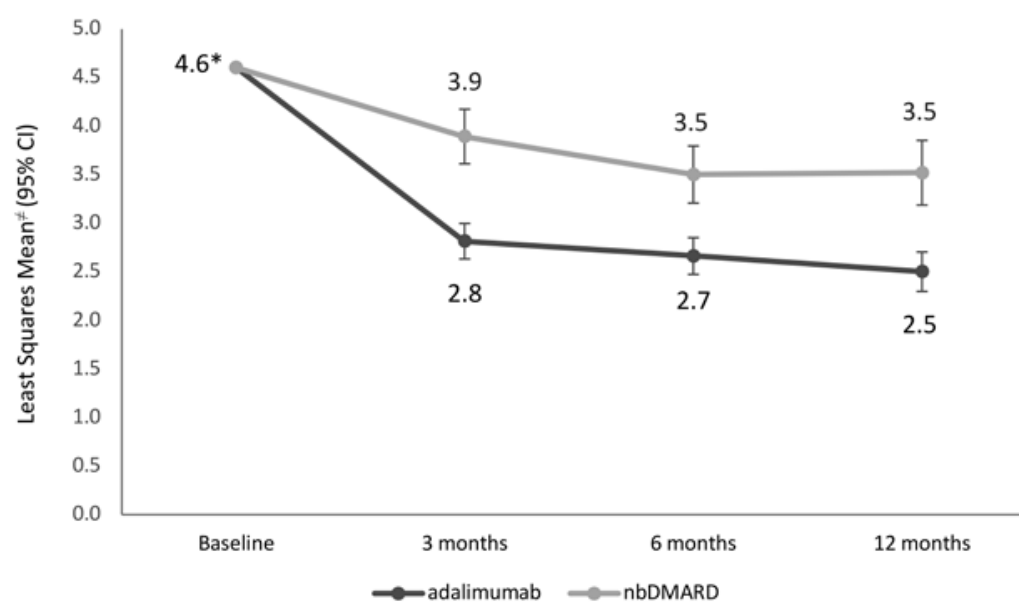

Figure 1. (A-E). ${ }^{\neq}$Represents adjusted means, specifically the least squares mean estimates derived from linear mixed-effects model with repeated measures, adjusting for baseline levels of the respective disease outcome, visit, and the interaction effect of treatment with visit. * Fixed at mean baseline levels. Bold $P$ values indicate statistical significance. CRP: C-reactive protein; DAPSA28: Disease Activity Index for Psoriatic Arthritis in 28 joints; DAS28: Disease Activity Score in 28 joints; ESR: erythrocyte sedimentation rate; nbDMARD: nonbiologic disease-modifying antirheumatic drug; PGA: physician global assessment.

included in the 3 studies. In addition to disease activity, treatment with ADA resulted in improved function over time, as well as significantly higher QOL at 6 months, a finding also corroborated by previous randomized clinical trials. ${ }^{7,80,22}$
A previous pooled analysis of randomized controlled trials demonstrated that biologic agents are effective in the treatment of dactylitis and enthesitis in patients with PsA. ${ }^{23}$ In our study, dactylitis among ADA-treated patients either resolved 


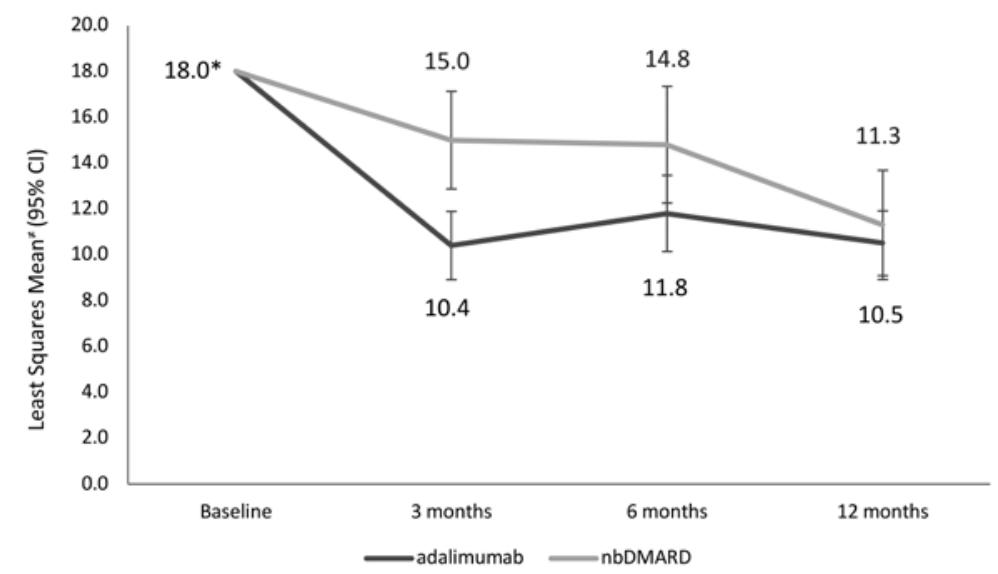

\section{CRP}

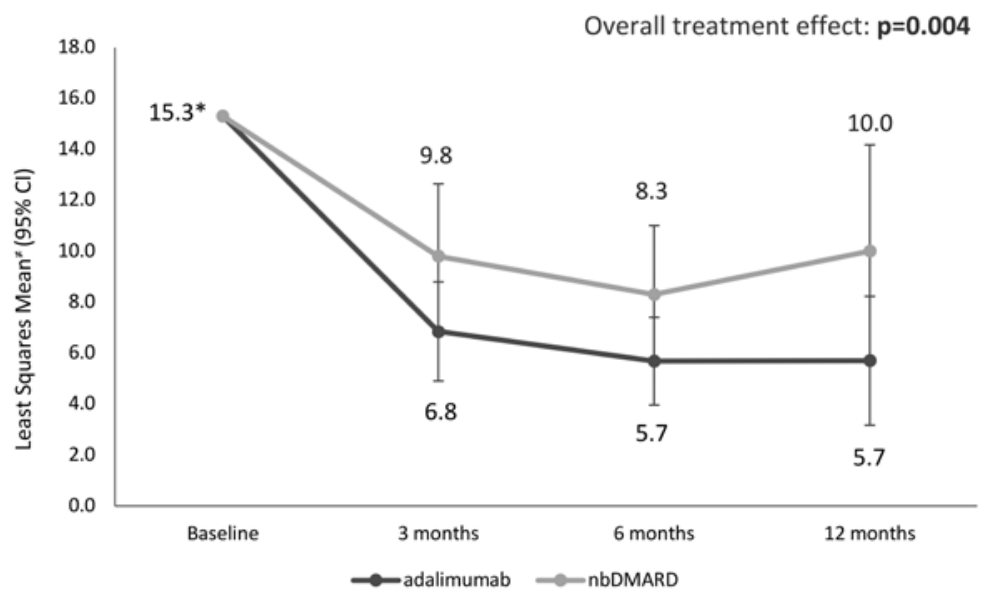

\section{E. PGA}

Overall treatment effect: $\mathbf{p}<0.001$

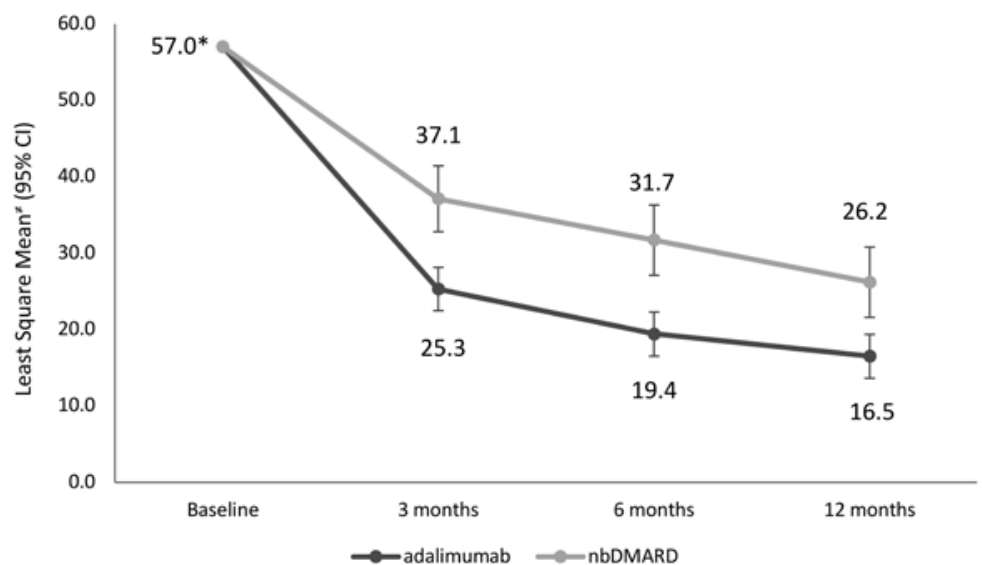

Figure 1. C-E.

or was significantly less likely to appear during the follow-up period. In fact, these patients were 2.5 times less likely to present with dactylitis during the follow-up period compared to nbDMARD-treated patients. However, there was no significant difference in the presence of enthesitis between treatment groups. While confirming the superiority of ADA in the treatment of dactylitis, these results suggest that $\mathrm{ADA}$ and nbDMARDs are equally as effective for the treatment of enthesitis. The severity of enthesitis was not measured in this study, and baseline enthesitis may have been more severe among patients in the ADA group, which would account for the lack of measured differences. Previous studies have also demonstrated that ADA is effective 


\section{A. PtGA}

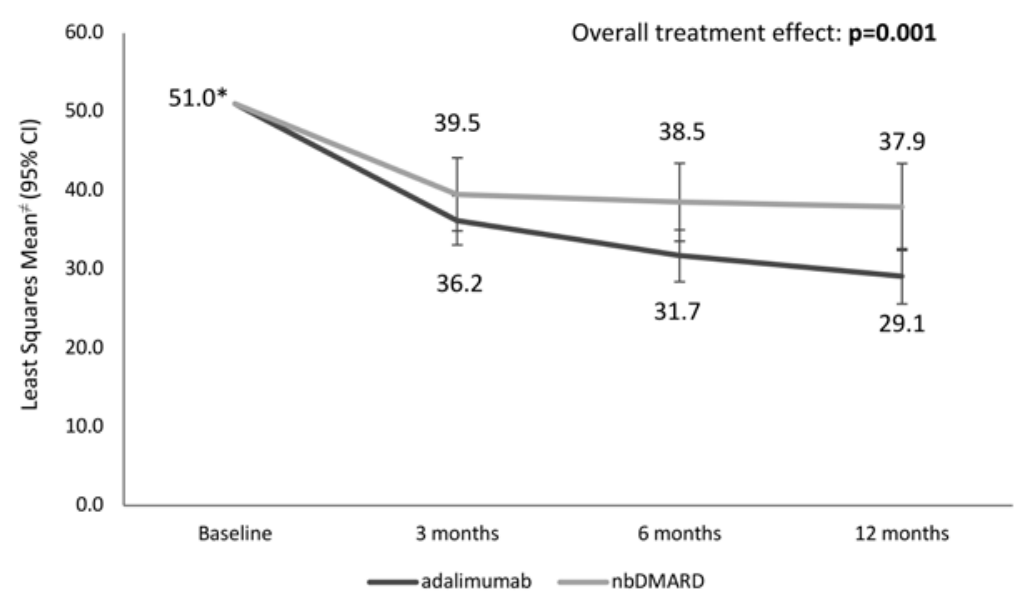

\section{B. HAQ-DI}

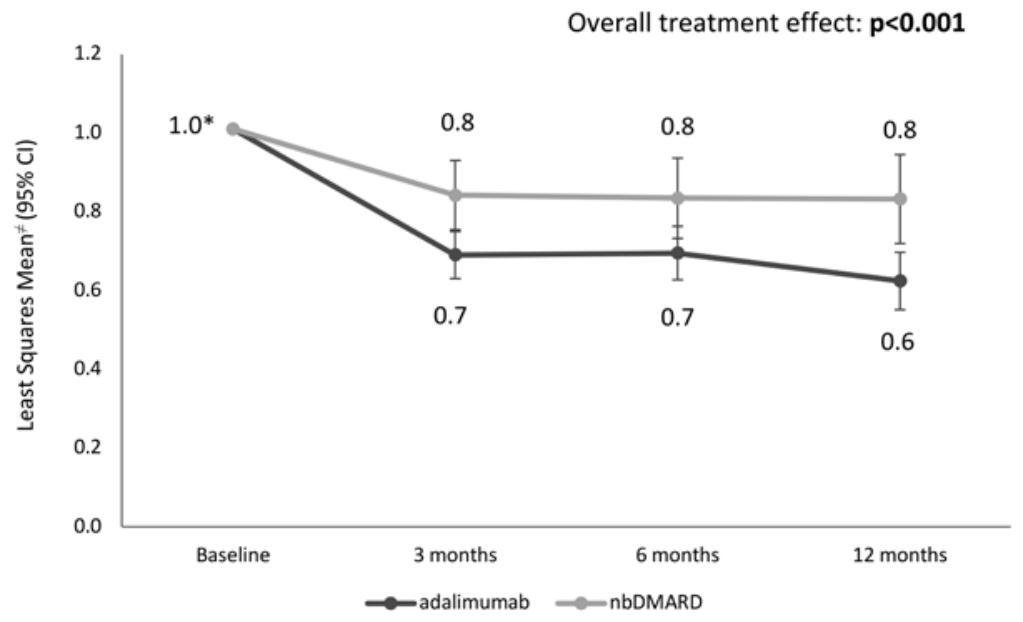

Figure 2. (A-E). ${ }^{*}$ Adjusted means, specifically the least squares mean estimates derived from linear mixed-effects model with repeated measures, adjusting for baseline levels of the respective disease outcome, visit, and the interaction effect of treatment with visit. ${ }^{*}$ Fixed at mean baseline levels. Bold $P$ values indicate statistical significance. SF-12 was not assessed at 3 months, as per the recommendations for follow-up assessments. HAQ-DI: Health Assessment Questionnaire-Disability Index; MCS: mental component summary; nbDMARD: nonbiologic disease-modifying antirheumatic drug; PCS: physical component summary; PtGA: patient global assessment; SF-12: 12-item Short Form Health Survey.

for the treatment of skin $\mathrm{PsO}_{\mathrm{S}}^{8,12,13,17,24}$ In this study, the odds of obtaining or maintaining mild $\mathrm{PsO}(\mathrm{BSA}<3 \%)$ was almost 3 times higher among patients treated with ADA.

With respect to treatment retention, patients in the ADA group were less likely to switch to a new biologic compared to patients in the nbDMARD group initiating a biologic agent. These results agree with previous studies in patients with PsA demonstrating that $\mathrm{ADA}$ treatment has a high rate of retention and is well tolerated over the long term. ${ }^{14,15,16}$ Safety findings, as assessed by the incidence of SAEs, were also consistent with the results of previous long-term investigations of ADA for the treatment of PsA. ${ }^{8,25}$

Our study has intrinsic limitations. Because of the nonrandomized nature of real-world studies, patients in our study selected for treatment with ADA had more severe baseline disease, suggesting a channeling bias. In an effort to account for these differences, multivariate models adjusting for potential baseline confounders were used. An additional limitation of the study is related to the fact that PsA was physician-diagnosed and not based on specific validated classification criteria. Regarding disease activity assessment, joint assessment was based on 28 joints instead of the full 66 of 68 joint assessment required for MDA assessment, and radiographic progression was not evaluated. It is also worth noting that only 2 entheseal points were evaluated, which may have resulted in an overestimation of the treatment effect. To counterbalance this, absence of enthesitis was used in calculating mMDA instead of the $\leq 1$ entheseal 


\section{Pain}

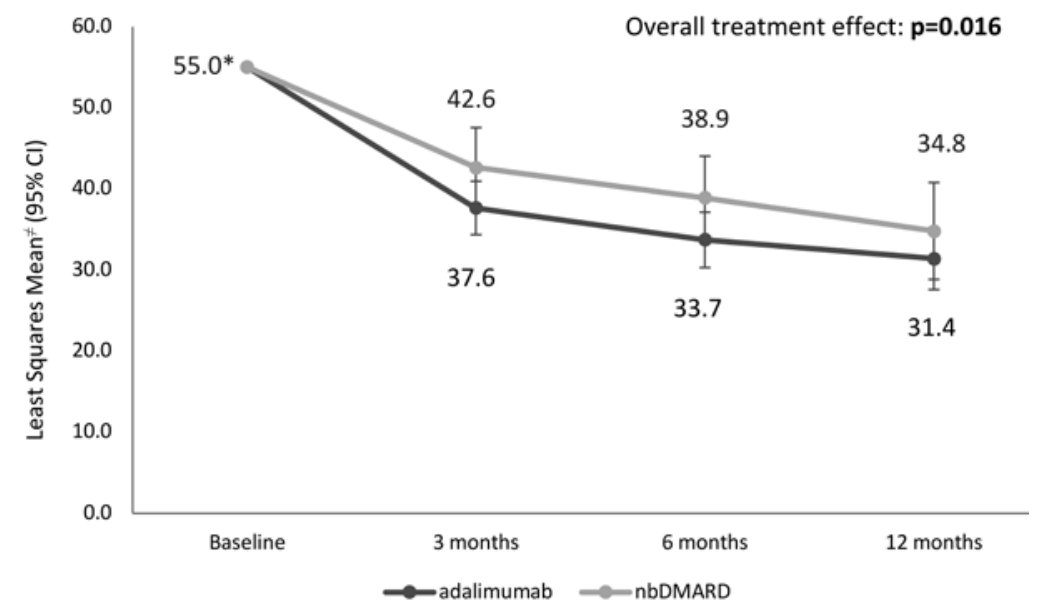

D. SF-12 (PCS)

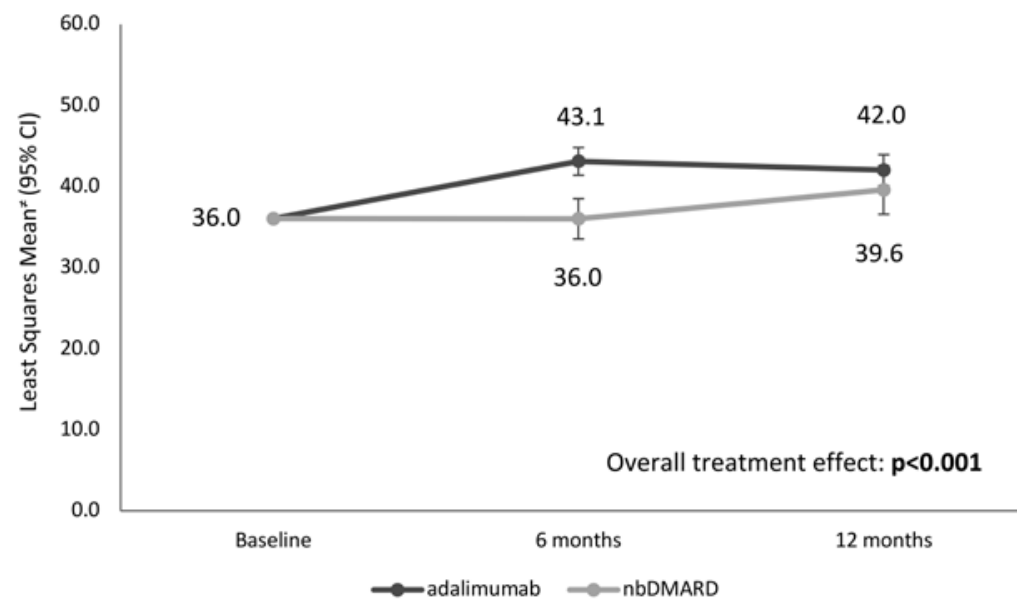

\section{E. SF-12 (MCS)}

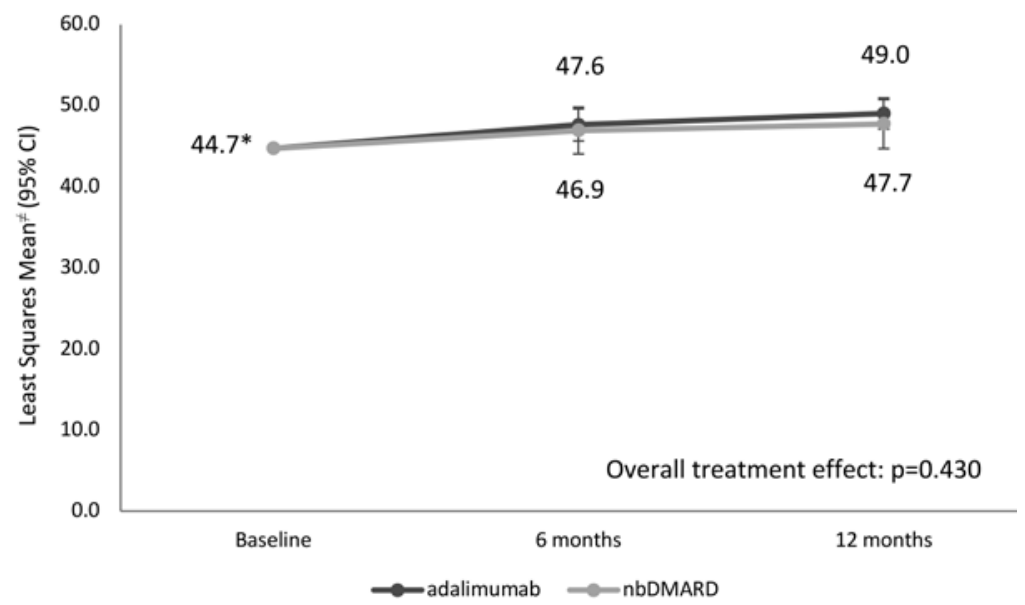

Figure 2. (C-E).

points included in the original MDA definition. In addition, systematic collection of SAEs only was required per the study protocol, thus not allowing a comprehensive analysis of safety.
Finally, the ADA treatment group in this study included both patients on monotherapy, as well as those treated with a combination nbDMARD regimen; this may be argued to have driven 
Table 2. Baseline-adjusted GEE model with repeated measures for enthesitis, dactylitis, and BSA over 12 months.

\begin{tabular}{lcc}
\hline & \multicolumn{2}{c}{ Treatment Group } \\
\cline { 2 - 3 } & \multicolumn{2}{c}{ Adalimumab vs nbDMARD } \\
\cline { 2 - 3 } & Adjusted ${ }^{\mathrm{a}}$ OR $(95 \% \mathrm{CI})$ & $P^{\mathrm{a}}$ \\
\hline Enthesitis (yes vs no) & $0.7(0.4-1.2)$ & 0.17 \\
Dactylitis (yes vs no) & $0.4(0.2-0.6)$ & $<0.001^{\mathrm{b}}$ \\
BSA affected (<3\% vs $\geq 3 \%)$ & $2.7(1.5-5.0)$ & $0.002^{\mathrm{b}}$ \\
\hline
\end{tabular}

${ }^{a}$ Adjusted for respective outcome at baseline. ${ }^{b}$ Statistically significant. BSA: body surface area; GEE: generalized estimating equations; OR: odds ratio; nbDMARD: nonbiologic disease-modifying antirheumatic drug.

the study results in favor of ADA. In order to address this, a sensitivity analysis was conducted assessing the differences between ADA combination- vs monotherapy-treated patients with respect to DAS28- and DAPSA28-related outcomes. As no statistically significant differences were found between groups, these findings have not been included in the current report.

The strengths of our study include the minimal inclusion criteria, a large sample size, and the management of patients per routine clinical practice, all of which enhance the external validity of our findings. Further, this study included several rheumatology clinics over a long follow-up period, allowing the inclusion of multiple new therapeutics and approaches to PsA management that have been introduced over time.

In conclusion, in this real-world study, ADA was more effective than nbDMARDs in reducing disease activity and severity of skin disease and in improving patient QOL, and was associated with significantly higher retention.

\section{ACKNOWLEDGMENT}

The authors wish to acknowledge JSS Medical Research for statistical analysis, medical writing, and editorial assistance during the preparation of this manuscript.

\section{ONLINE SUPPLEMENT}

Supplementary material accompanies the online version of this article.

\section{REFERENCES}

1. Neimann AL, Porter SB, Gelfand JM. The epidemiology of psoriasis. Expert Rev Dermatol 2006;1:63-75.

2. Gladman DD, Antoni C, Mease P, Clegg DO, Nash P. Psoriatic arthritis: epidemiology, clinical features, course, and outcome. Ann Rheum Dis 2005;64 Suppl 2:ii14-7.

3. Gladman DD. Clinical aspects of the spondyloarthropathies. Am J Med Sci 1998;316:234-8.

4. Singh JA, Guyatt G, Ogdie A, et al. Special article: 2018 American College of Rheumatology/National Psoriasis Foundation guideline for the treatment of psoriatic arthritis. Arthritis Care Res 2019; 71:2-29.

5. Coates LC, Kavanaugh A, Mease PJ, et al. Group for Research and Assessment of Psoriasis and Psoriatic Arthritis 2015 treatment recommendations for psoriatic arthritis. Arthritis Rheumatol 2016;68:1060-71.

6. Government of Canada. Notice of compliance search engine. [Internet. Accessed January 11, 2022.] Available from: https:// health-products.canada.ca/noc-ac
7. Genovese MC, Mease PJ, Thomson GT, et al. Safety and efficacy of adalimumab in treatment of patients with psoriatic arthritis who had failed disease modifying antirheumatic drug therapy. J Rheumatol 2007;34:1040-50.

8. Gladman DD, Mease PJ, Ritchlin CT, et al. Adalimumab for long-term treatment of psoriatic arthritis: forty-eight week data from the adalimumab effectiveness in psoriatic arthritis trial. Arthritis Rheum 2007;56:476-88.

9. Boehncke WH, Alvarez Martinez D, Solomon JA, Gottlieb AB. Safety and efficacy of therapies for skin symptoms of psoriasis in patients with psoriatic arthritis: a systematic review. J Rheumatol 2014;41:2301-5.

10. Mease PJ, Ory P, Sharp JT, et al. Adalimumab for long-term treatment of psoriatic arthritis: 2-year data from the Adalimumab Effectiveness in Psoriatic Arthritis Trial (ADEPT). Ann Rheum Dis 2009;68:702-9.

11. Saurat JH, Stingl G, Dubertret L, et al. Efficacy and safety results from the randomized controlled comparative study of adalimumab vs. methotrexate vs. placebo in patients with psoriasis (CHAMPION). Br J Dermatol 2008;158:558-66.

12. Menter A, Tyring SK, Gordon K, et al. Adalimumab therapy for moderate to severe psoriasis: a randomized, controlled phase III trial. J Am Acad Dermatol 2008;58:106-15.

13. Carrera CG, Dapavo P, Malagoli P, et al. PACE study: real-life Psoriasis Area and Severity Index (PASI) 100 response with biological agents in moderate-severe psoriasis. J Dermatolog Treat 2018;29:481-6.

14. Fagerli KM, Kearsley-Fleet L, Watson KD, et al. Long-term persistence of TNF-inhibitor treatment in patients with psoriatic arthritis. Data from the British Society for Rheumatology Biologics Register. RMD Open 2018;4:e000596.

15. Iannone F, Lopriore $S$, Bucci R, et al. Longterm clinical outcomes in 420 patients with psoriatic arthritis taking anti-tumor necrosis factor drugs in real-world settings. J Rheumatol 2016;43:911-7.

16. Saad AA, Ashcroft DM, Watson KD, et al. Persistence with anti-tumour necrosis factor therapies in patients with psoriatic arthritis: observational study from the British Society of Rheumatology Biologics Register. Arthritis Res Ther 2009;11:R52.

17. Zweegers J, Groenewoud JM, van den Reek JM, et al. Comparison of the 1- and 5-year effectiveness of adalimumab, etanercept and ustekinumab in patients with psoriasis in daily clinical practice: results from the prospective BioCAPTURE registry. Br J Dermatol 2017;176:1001-9.

18. Michelsen B, Sexton J, Smolen JS, et al. Can disease activity in patients with psoriatic arthritis be adequately assessed by a modified Disease Activity Index for PSoriatic Arthritis (DAPSA) based on 28 joints? Ann Rheum Dis 2018;77:1736-41.

19. Heiberg MS, Kaufmann C, Rødevand E, et al. The comparative effectiveness of anti-TNF therapy and methotrexate in patients with psoriatic arthritis: 6 month results from a longitudinal, observational, multicentre study. Ann Rheum Dis 2007; 66:1038-42.

20. Gladman DD, ACCLAIM Study Investigators, Sampalis JS, Illouz $\mathrm{O}$, Guérette B. Responses to adalimumab in patients with active psoriatic arthritis who have not adequately responded to prior therapy: effectiveness and safety results from an open-label study. J Rheumatol 2010;37:1898-906.

21. Van den Bosch F, Manger B, Goupille P, et al. Effectiveness of adalimumab in treating patients with active psoriatic arthritis and predictors of good clinical responses for arthritis, skin and nail lesions. Ann Rheum Dis 2010;69:394-9.

22. Mease PJ, Gladman DD, Ritchlin CT, et al. Adalimumab for the treatment of patients with moderately to severely active psoriatic arthritis: results of a double-blind, randomized, placebo-controlled trial. Arthritis Rheum 2005;52:3279-89. 


\section{A. ACR50}

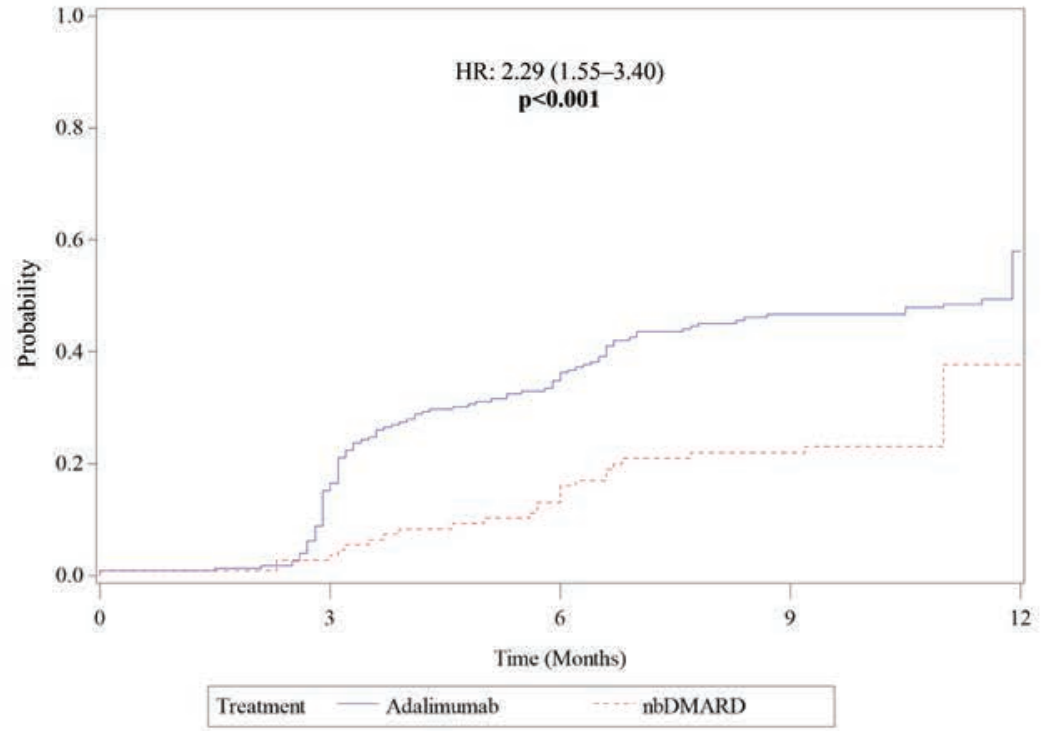

\begin{tabular}{|c|c|c|}
\hline \multirow{2}{*}{ Month } & \multicolumn{2}{|c|}{$\begin{array}{c}\text { Cumulative probability of } \\
\text { achieving ACR50 }\end{array}$} \\
\hline & Adalimumab & nbDMARD \\
\hline 3 & $15.2 \%(11.1 \%-20.6 \%)$ & $2.8 \%(0.9 \%-8.3 \%)$ \\
\hline 6 & $34.9 \%(29.0 \%-41.6 \%)$ & $13.1 \%(8.0 \%-21.2 \%)$ \\
\hline 12 & $57.9 \%(50.6 \%-65.4 \%)$ & $37.8 \%(27.7 \%-50.1 \%)$ \\
\hline
\end{tabular}

B. ACR70

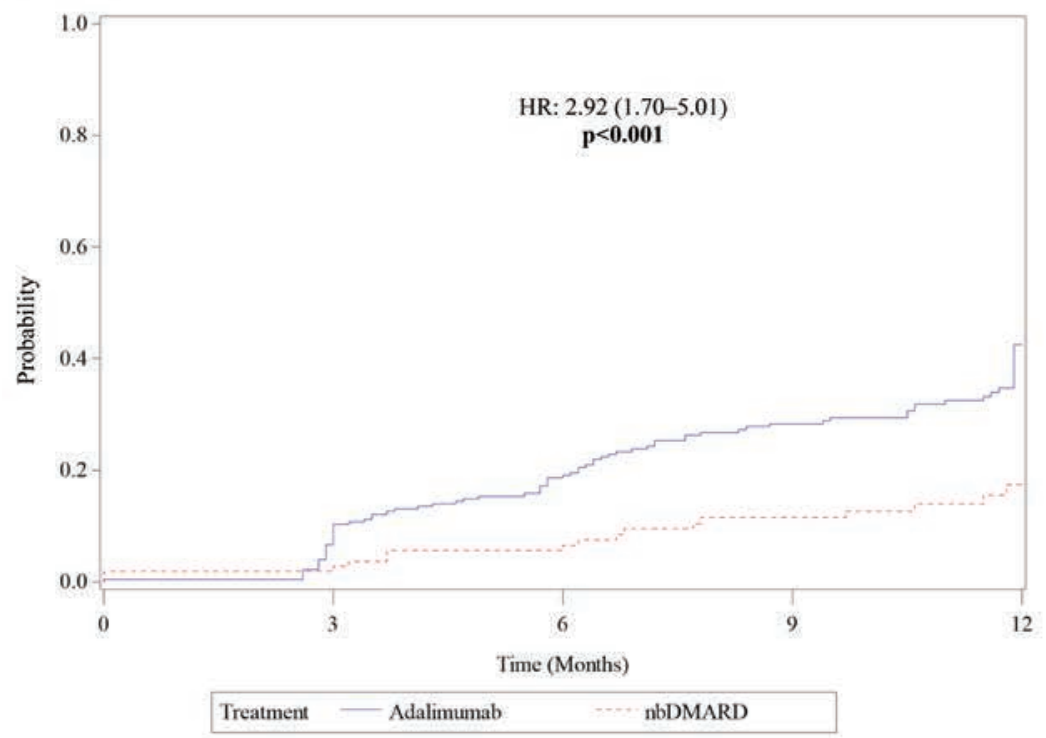

\begin{tabular}{|c|c|c|}
\hline Month & \multicolumn{2}{|c|}{$\begin{array}{c}\text { Cumulative probability of } \\
\text { achieving ACR70 }\end{array}$} \\
\hline & Adalimumab & nbDMARD \\
\hline 3 & $6.7 \%(4.1 \%-10.9 \%)$ & $1.8 \%(0.5 \%-7.1 \%)$ \\
\hline 6 & $18.6 \%(14.1 \%-24.4 \%)$ & $5.6 \%(2.6 \%-12.0 \%)$ \\
\hline 12 & $42.4 \%(35.3 \%-50.4 \%)$ & $17.4 \%(10.8 \%-27.7 \%)$ \\
\hline
\end{tabular}

Figure 3. (A-C). ${ }^{\dagger}$ MDA based on a modified measure, which required the presence of 5 out of the 7 following outcomes: TJC $\leq 1$, SJC $\leq 1, \mathrm{BSA} \leq 3 \%$, pain VAS $\leq 15$, PtGA $\leq 20$, HAQ-DI $\leq 0.5$, and absence of enthesitis. Bold $P$ values indicate statistical significance. ACR50/70: American College of Rheumatology $50 \%$ or $70 \%$ response; BSA: body surface area; HAQ-DI: Health Assessment Questionnaire-Disability Index; HR: hazard ratio; MDA: minimal disease activity; mMDA: modified minimal disease activity; nbDMARD: nonbiologic disease-modifying antirheumatic drug; NSAID: nonsteroidal antiinflammatory drug; PtGA: patient global assessment; SJC: swollen joint count; TJC: tender joint count; VAS: visual analog scale. 


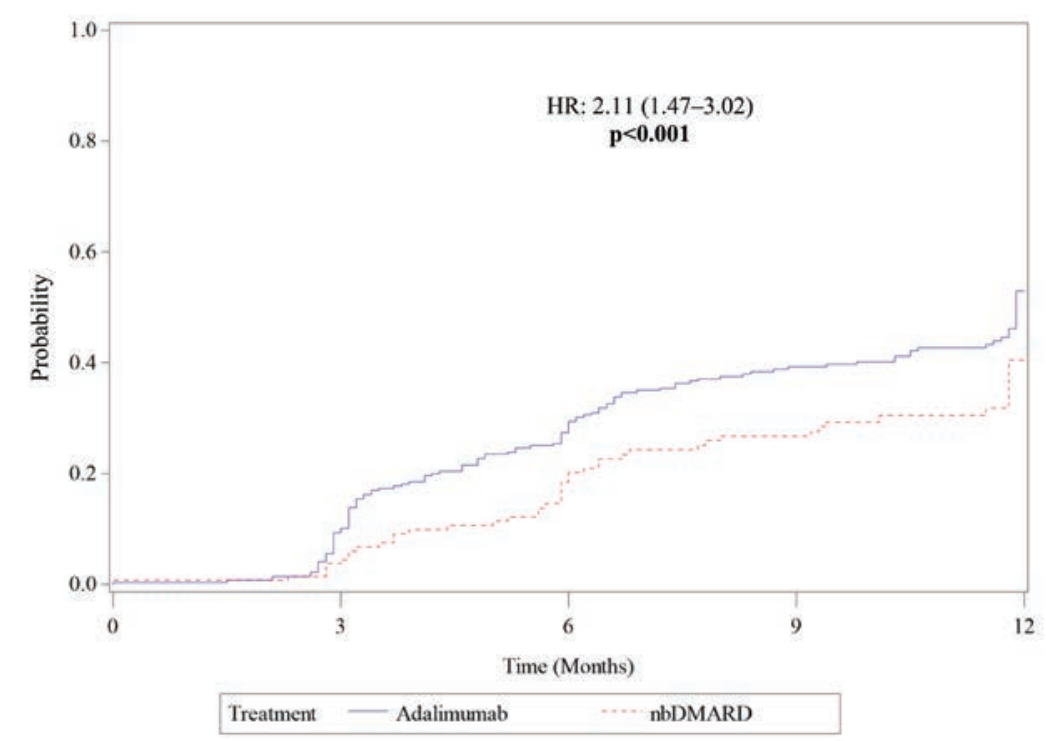

\begin{tabular}{|c|c|c|}
\hline Month & \multicolumn{2}{|c|}{$\begin{array}{r}\text { Cumulative probability of } \\
\text { achieving mMDA }\end{array}$} \\
\hline & Adalimumab & nbDMARD \\
\hline 3 & $9.3 \%(6.4 \%-13.5 \%)$ & $3.7 \%(1.6 \%-8.7 \%)$ \\
\hline 6 & $27.4 \%(22.4 \%-33.2 \%)$ & $18.6 \%(12.8 \%-26.4 \%)$ \\
\hline 12 & $52.9 \%(46.1 \%-60.1 \%)$ & $40.5 \%(31.5 \%-51.0 \%)$ \\
\hline
\end{tabular}

Figure 3. (C).

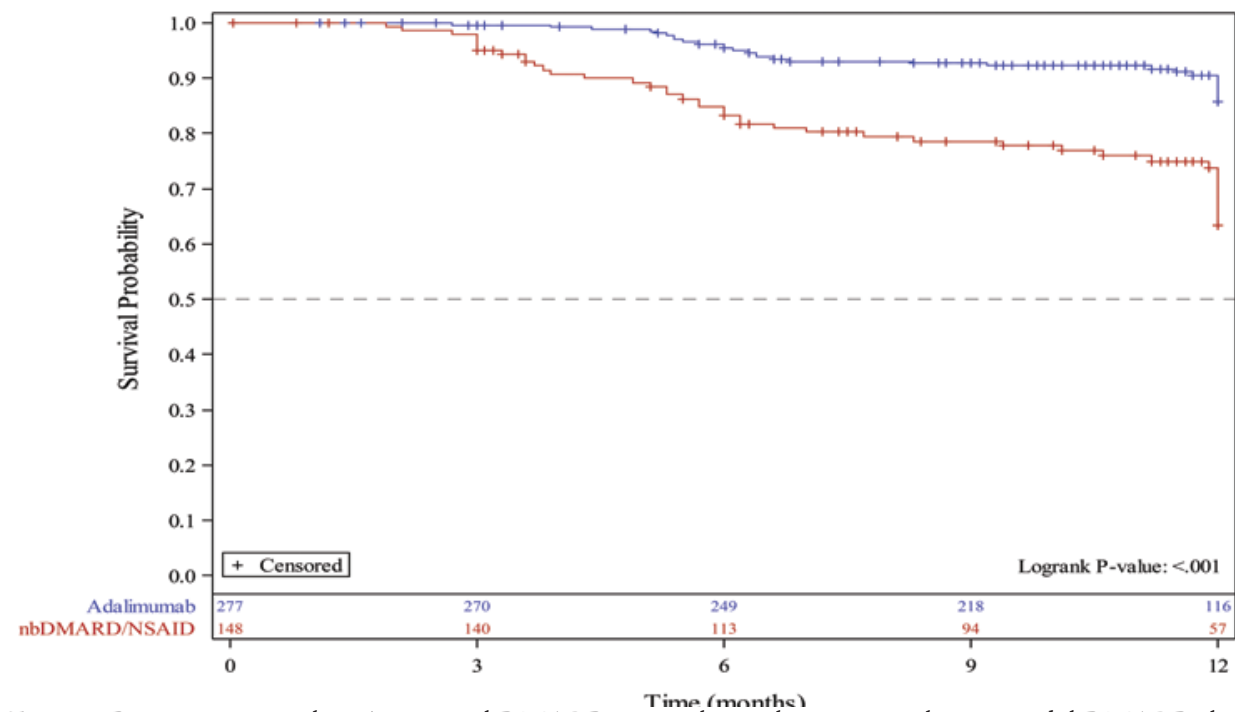

Figure 4. Patients not switching/initiating bDMARD up until month 12 were right censored. bDMARD: biologic disease-modifying antirheumatic drug; nbDMARD: nonbiologic disease-modifying antirheumatic drug; NSAID: nonsteroidal antiinflammatory drug.

23. Mourad A, Gniadecki R. Treatment of dactylitis and enthesitis in psoriatic arthritis with biologic agents: a systematic review and metaanalysis. J Rheumatol 2020;47:59-65.

24. López-Ferrer A, Vilarrasa E, Gich IJ, Puig L. Adalimumab for the treatment of psoriasis in real life: a retrospective cohort of 119 patients at a single Spanish centre. Br J Dermatol 2013;169:1141-7.

25. Burmester GR, Gordon KB, Rosenbaum JT, et al. Long-term safety of adalimumab in 29,967 adult patients from global clinical trials across multiple indications: an updated analysis. Adv Ther 2020;37:364-380. 